\title{
Reply to a comment on "Quantum theory and the limits of objectivity"
}

\author{
Richard Healey*
}

\begin{abstract}
In this short note I reply to criticisms of an argument in my paper [1] that appear in comment [2]. I refer the reader to section 4 of [1] in which I described the scenario of a Gedankenexperiment on which is based the argument criticized in [2]. The authors of 2] raise one "main criticism" then go on to claim that the argument of [1] contains a series of problems. But their "main criticism" is not an objection to the argument and the problems are of their own making. In replying to what they call their main criticism I will take this opportunity to exhibit the structure of the argument and so make clear why this is not an objection to that argument.
\end{abstract}

In this short note I reply to criticisms of an argument in my paper [1] that appear in comment [2]. I refer the reader to section 4 of [1] in which I described the scenario of a Gedankenexperiment on which is based the argument criticized in 2]. The authors of 2] raise one "main criticism" then go on to claim that the argument of [1] contains a series of problems. But their "main criticism" is not an objection to the argument and the problems are of their own making. In replying to what they call their main criticism I will take this opportunity to exhibit the structure of the argument and so make clear why this is not an objection to that argument.

I begin by addressing the "main criticism" offered in [2, there stated as follows:

"...the computed correlation functions entering the Bell's inequality are in principle experimentally inaccessible, and hence the author's claim is in principle not testable".

There is a simple reply. To regard this as a criticism of the argument in section 4 of [1] is to misunderstand the structure of that argument. That the probabilistic correlations predicted by quantum theory in the scenario of the Gedankenexperiment there described are not (in a certain sense) testable is a feature of the argument, not a bug! That argument proceeds by reductio ad absurdum. It proves that a number of plausible premises are in fact mutually inconsistent. If the proof is valid then at least one of these premises must be

${ }^{*}$ Philosophy Department, University of Arizona, Tucson, AZ 85721, USA and Stellenbosch Institute for Advanced Study (STIAS), Wallenberg Research Centre at Stellenbosch University, Stellenbosch 7600, South Africa. 
false. The point of the argument is to explore the consequences for objectivity of the assumption that the probabilistic predictions of unitary quantum theory are correct, including when the theory is applied to an entire isolated experimental laboratory containing an experimenter performing a quantum measurement and recording its outcome. Whether these predictions can be tested in the scenario of the Gedankenexperiment is simply irrelevant to the validity of the argument.

Here is the sense in which the probabilistic correlation functions predicted by quantum theory in the scenario of the Gedankenexperiment described in section 4 of [1] are untestable. Frequency data of the outcomes of every repetition of the sequence of single quantum measurements by Alice, Bob, Carol and Dan could not all be tabulated together in a single, localized space-time region within the world-tube of a single spatially-localized experimenter. So no localized agent (not just Alice, Bob, Carol or Dan) could compare the statistical correlation functions computed from these data with the probabilistic correlation functions predicted by quantum theory to see how well they match. I take it that this is what is meant by the first sentence of the passage from [2] describing the "first problem" with the argument of [1:

"The violation of the proposed Bell's inequality cannot, not even in principle, be tested, because in no region of space-time are the experimental data from which all the correlations functions can be extracted available."

Of course, the assumption (A) implies that all this experimental data is present somewhere in the much larger space-time region in which are located all the quantum measurements performed throughout the duration of the Gedankenexperiment.

(A) Every quantum measurement has a definite (unique, objective) physical outcome.

But, though (by assumption) present there, all this data is not epistemically accessible to any individual experimenter. Any attempt by an experimenter to access all this data would involve physical interactions that would break the isolation of the experimenters' labs involved in the Gedankenexperiment and disrupt the delicate sequence of operations they perform in each repetition wiithin the Gedankenexperiment.

That the violation of the proposed Bell's inequality cannot, not even in principle, be tested raises no problem for the argument of [1].

The authors of 2 attempt to raise a second problem that would be faced by anyone wishing to remove the alleged first problem by "attributing an operational meaning to the computed expressions for the [probabilistic] correlation functions". But this is no problem, since, having dismissed the first "problem", anyone giving the argument of [1] has no need to "attribute an operational meaning to the computed correlation functions" and should not wish to do so.

As the authors of [2] correctly note, if Alice and Bob were to try to test their predictions and actually violate a Bell's inequality with data they had collected, they would have to adapt the experimental protocol in some way (which then may or may not in fact predict such violation). But the validity of the argument of [1] neither requires nor motivates any change in the protocol there described. As it stands, that argument does not require "the standard 
assumptions for testing the violation of Bell's inequalities namely 'freedom of choice' and 'locality"'.

In a section entitled "Analysis of the protocol" the authors of [2] discuss what they call "fundamental problems with the proposal in [1]". After repeating the (true, but irrelevant) claim of untestability, they diagnose "multiple problems" stemming from the assumption that the measurement of Carol and Dan are considered as unitary transformations. Now it is a basic assumption of the entire reductio argument that every interaction may be viewed as proceeding in accordance with a unitary transformation in the relevant total quantum state. The argument shows that this assumption leads to conflict with assumption (A)-that was the point of the argument, not a problem with it.

The first of the "multiple problems" concerns an ambiguity that allegedly arises from the fact that certain probabilistic correlation functions are computed from two different reference frames. The authors of 2 note that that there would be no ambiguity if these correlation functions would refer to measurement data, since these are reference frame independent. But the correlation functions do refer to measurement data: in the correlation $E(a, d)$ they refer to data collected in measurements by Alice and by Dan, while in the correlation $E(b, c)$ they refer to data collected in measurements by Bob and by Carol. In a single repetition within the Gedankenexperiment these data are collected in the regions of Figure 1 of [1] there labeled $U_{A}^{1}, U_{D}^{2}, U_{B}^{2}, U_{C}^{1}$ respectively. Assumption (A) guarantees the objectivity of this data, even while each of their measurement interactions is assumed to correspond to the unitary transformation used to label these regions of the figure.

The second of these "problems" alleges another kind of ambiguity, of a kind first alleged in [3]. Alternative ways of calculating $E(a, d)$ and $E(b, c)$ are proposed, in each case leading to an alternative value for this quantity: specifically, evaluated in Alice's reference frame $E(b, c)=0$ for all times rather than $-\cos (b-c)$ as claimed in [1. But this evaluation in Alice's frame is incorrect, since it assumes that it is possible to assign a value to the outcome of a measurement by Bob (Carol) even though Bob's (Carol's) register is in, or returned to, its fixed pre-measurement state. The only correlations considered in the argument of [1] are correlations between the measurement outcomes whose objective occurrence follows from assumption (A). Each of these outcomes occurs in the localized space-time region where the relevant measurement is performed. No outcome occurs in a region where a register is in its pre-measurement state: and a measurement by Carol (Dan) occurs when and where she (he) makes it, not in a space-time region where Alice or Bob might have performed a measurement on Carol's (Dan's) lab to try to inspect their outcomes. This second problem does not arise for the reason the authors of 2] themselves state:

"If the four measurements would be identified with four space-time points in which counts are registered, then the correlations between these counts will be reference-frame independent and there would be no problem." The "main criticism" made in 2] of the argument in section 4 of [1] leaves that argument untouched, and, on careful examination, the "multiple problems" alleged in [2] turn out not to be problems at all. 
Acknowledgement 1 This note was composed while I was a Fellow of the Stellenbosch Institute for Advanced Studies: I am grateful to STIAS for their support.

\section{References}

[1] Healey, R.: Quantum theory and the limits of objectivity. Found. Phys. 48(11). 1568-89 (2018).

[2] Baumann, V., Del Santo, F., and Brukner, C..: Comment on Healey's "Quantum theory and the limits of objectivity". Found. Phys. Published online: 12 June 2019.

[3] Gao, S.: Unitary quantum theory is incompatible with special relativity. (2018). Preprint https://philpapers.org/rec/GAOUQT. 